\title{
Energy resolved X-ray grating interferometry
}

\author{
T. Thuering, ${ }^{1,2}$ W. C. Barber, ${ }^{3}$ Y. Seo, ${ }^{4}$ F. Alhassen, ${ }^{4}$ J. S. Iwanczyk, ${ }^{3}$ and M. Stampanoni ${ }^{1,2}$ \\ ${ }^{1}$ Swiss Light Source, Paul Scherrer Institut, Villigen PSI, Switzerland \\ ${ }^{2}$ Institute for Biomedical Engineering, Swiss Federal Institute of Technology, Zurich, Switzerland \\ ${ }^{3}$ DxRay, Inc., Northridge, California 91324, USA \\ ${ }^{4}$ UCSF Physics Research Laboratory, Department of Radiology and Biomedical Imaging, \\ University of California, San Francisco, California 94143, USA
}

(Received 4 March 2013; accepted 1 May 2013; published online 15 May 2013)

\begin{abstract}
Although compatible with polychromatic radiation, the sensitivity in X-ray phase contrast imaging with a grating interferometer is strongly dependent on the X-ray spectrum. We used an energy resolving detector to quantitatively investigate the dependency of the noise from the spectral bandwidth and to consequently optimize the system-by selecting the best energy band matching the experimental conditions-with respect to sensitivity maximization and, eventually, dose. Further, since theoretical calculations of the spectrum are usually limited due to non-ideal conditions, an energy resolving detector accurately quantifies the spectral changes induced by the interferometer including flux reduction and beam hardening. (C) 2013 AIP Publishing LLC. [http://dx.doi.org/10.1063/1.4805073]
\end{abstract}

Exploiting X-ray phase or scattering instead of conventional absorption based imaging can yield complementary information or enhanced image contrast. ${ }^{1,2}$ Using a grating interferometer (GI), this has been demonstrated for a variety of applications, as, for instance, in biomedical imaging or materials science. ${ }^{1,3,4}$ The possibility of using grating interferometry on conventional X-ray sources ${ }^{5,6}$ opened the path towards mainstream applications on table top scanners. ${ }^{7,8}$ However, the performance of the GI using conventional $\mathrm{X}$-ray sources is limited due to the polychromatic radiation. The sensitivity of the GI in polychromatic mode is still not very well understood, neither analytically nor experimentally. Here, the effects of the gratings to the X-ray spectrum from a tungsten tube as well as the dependency of the sensitivity of the GI from the spectrum is experimentally investigated by using an energy resolving detector. In addition, the optimization of the sensitivity for grating based differential phase contrast (DPC) imaging by using a selective spectral bandwidth limitation is demonstrated.

Energy resolved detection implies an energy dependent selection of the X-rays, also referred to as energy binning. Single photon counting detectors with selective energy thresholding provide this feature by counting a photon if its energy is higher or equal than the selected threshold value. By acquiring data with multiple thresholds in parallel or by performing multiple acquisitions with different thresholds (threshold sweep), the number of photons as a function of the threshold value can be measured in each pixel. By calculating the derivative of this function with respect to the threshold value yields the number of photons per energy bin, whereas the bin size is given by the difference of two consecutive threshold values. This allows measurements of the X-ray spectrum or a retrieval of radiographic and tomographic X-ray images in each energy bin separately (energy resolved imaging).

Standard for today's single photon counting imaging detectors are silicon sensors for the direct conversion of $\mathrm{X}$-rays into an electrical signal. However, at energies above approx. $20 \mathrm{keV}$, which is typical for grating interferometry, the efficiency of such sensors decays dramatically. For the herein presented experiments, a custom cadmium telluride (CdTe) based solid state imaging array was used with a nearly ideal efficiency for X-ray energies of up to $80 \mathrm{keV}$. The array has four rows of 64 pixels with a $1.1 \mathrm{~mm}$ pitch formed by depositing a pixellated metalized anode pattern on one side of $1 \mathrm{~mm}$ thick CdTe single crystals. ${ }^{9}$ A continuous metal cathode is deposited on the other side of the crystals and a $-500 \mathrm{~V}$ bias voltage is applied. The pixels are connected to the inputs of eight 32 channel low noise application specific integrated circuits (ASICs) optimized for high flux energy dispersive X-ray single photon counting. Each parallel channel contains a fast peaking and shaping stage which is connected to four parallel threshold discriminators. A digital counter iterates up by one for each pulse above an individual threshold setting. Data can be acquired in frames as short as $1 \mathrm{~ms}$ and the parallel architecture of the ASIC allows a full 1000 frames per second readout. The count rate response is linear to $400 \mathrm{kcps}$ per pixel and has a maximum of 1 Mcps. With a pixel size of $1.1 \mathrm{~mm}$, the detector as such is not compatible with microscopic imaging applications, but would have a great potential for medical imaging as for instance computed tomography (CT). The purpose of our experiment is to highlight the potential impact of such a detector for energy resolved phase contrast imaging with a GI.

A symmetric Talbot-Lau type interferometer, consisting of a source grating (G0), a beam splitter (G1), and an analyzer grating $(\mathrm{G} 2)$, has been set up for this experiment. The grating periods are $p_{0}=p_{1}=p_{2}=2.4 \mu \mathrm{m}$ and the grating distances, G0-to-G1 and G1-to-G2, are both $100 \mathrm{~mm}$. The $\mathrm{X}$-ray source is a microfocus tube with a tungsten target, operating at a voltage of $50 \mathrm{kV}$ and a current of $50 \mu \mathrm{A}$. G0 is an absorption grating of gold lines generating a periodic structure of transmitting and opaque lines. Each individual transmitting slit of G0 forms an X-ray source providing enough spatial coherence required to generate interference. ${ }^{5}$ The interference pattern itself is generated by $\mathrm{G1}$, which is a 
phase grating with Nickel lines, periodically shifting the phase by zero and $\pi$ at the design energy $E_{d}=28 \mathrm{keV}$. At the position of $\mathrm{G} 2$, the intensity of the interference pattern is approximately a sine of period $p_{2}$. G2 is an absorption grating with gold lines, similar as G0; however, it is used to detect transverse shifts in the intensity pattern caused by the sample. All gratings are fabricated on titanium substrates with a thickness of $50 \mu \mathrm{m}$ for G0 and G1 and $140 \mu \mathrm{m}$ for G2. Due to the compact design, the gratings are curved to maintain a large field of view.

Energy resolving detectors allow the assessment of the $\mathrm{X}$-ray spectrum impinging on the detector, which is essential to analyze the performance of the GI. Gratings and substrates attenuate the beam and alter the shape of the spectrum. Fig. 1 shows a measurement of the beam spectrum at the detector for different grating configurations. The data were acquired by using a threshold sweep scan with an exposure time of $5 \mathrm{~s}$ per sweep step. The highest loss of photons occurs after inserting G0. Inserting G1 has no strong effect, since the Nickel lines are highly transparent and most of the photons, especially at low energies, have already been filtered out by G0. G2 again attenuates a larger fraction, which is due to the thicker substrate and the high absorbing gold structures. In addition to the spectra, Fig. 1(b) shows the energy resolved transmission of the $\mathrm{X}$-rays, indicating a maximum attenuation of photons at around $18 \mathrm{keV}$. The transmission through all gratings, which is given by the ratio of the integrals of the spectra, is $26 \%$. This would correspond to an increase in scan time by a factor of 3.7 compared to standard absorption imaging without any gratings. Another important factor which can be extracted from these data is the intrinsic increase of the required dose for an absorption image with the GI to achieve the same signal-to-noise ratio (SNR) as with a standard absorption imaging setup without a GI. The required increase of dose is a result of the post sample attenuation of the X-rays which occurs because the object is normally placed in front of G1 and G2. Fig. 1(b) shows the energy resolved X-ray transmission through G1 and G2 only. The total transmission through G1 and G2 is $47 \%$, which indicates that a dose increase by a factor of $\sqrt{1 / 0.47}=1.46$ would be necessary for the GI to obtain the same SNR as for an absorption image acquired without the GI. This factor matches very well with theoretical predictions. $^{10,11}$

The attenuation in the gratings and substrates further introduces some beam hardening, changing the mean energy of the spectrum. In Fig. 1(a), the mean energies are indicated with the vertical lines for each spectrum. The maximum shift in energy from the setup without gratings to the setup with all gratings is $4.2 \mathrm{keV}$. Since the GI is designed for a mean energy of $28 \mathrm{keV}$, any change in the mean energy or in the width of the spectrum is affecting the sensitivity of the GI through a change of the visibility. The visibility is defined as $V=2\left|a_{1}\right| /\left|a_{0}\right|$, where $a_{0}$ and $a_{1}$ are the zeroth and first Fourier coefficients, respectively, of the phase stepping curve. ${ }^{12}$ Since phase contrast images are recorded through a measurement of the refraction angle, the sensitivity of the GI is here defined as the noise standard deviation of the refraction angle $\alpha$, given by ${ }^{13}$

$$
\sigma_{\alpha}=\frac{p_{2}}{2 \pi d} \frac{\sqrt{2 \chi_{\mathrm{det}}}}{V \sqrt{N}}
$$

where $p_{2}$ is the pitch of $\mathrm{G} 2, d$ is the distance between $\mathrm{G} 1$ and G2, $V$ is the visibility, $N$ is the number of photons (or detected ADU number), and $\chi_{\text {det }}$ is a detector specific parameter, which relates the detected quanta and the detector noise variance by $\sigma_{\mathrm{det}}^{2}=\chi_{\mathrm{det}} N$.

Equation (1) shows that the sensitivity of a phase contrast image is strongly dependent on the visibility $V$. Therefore, the performance of a GI can be analyzed by evaluating the visibility as a function of the (monochromatic) $\mathrm{X}$-ray energy. ${ }^{14} \mathrm{Fig}$. 2(a) shows such a curve for the experimental setup used here, obtained by a computer simulation based on wave optics. The spectral acceptance, here defined as the energy bandwidth around the design energy and limited by the zero crossings of the visibility, ranges from 21 to $42 \mathrm{keV}$. Using an energy resolving detector, this curve can be determined experimentally. A phase stepping scan, ${ }^{12}$ combined with a threshold sweep over a range between 10 and $50 \mathrm{keV}$, has been performed on the experimental setup, using an energy bin size of $3 \mathrm{keV}$ (sliding window). Fig. 2(b) shows the result of this scan. For a comparison, this curve has also been simulated for the given source, grating and detector parameters (dashed line in the figure). The dominating peak of the visibility located at approx. $29 \mathrm{keV}$ coincides very well with the design energy $E_{d}=28$ of the interferometer. The error of approx. $1 \mathrm{keV}$ is most likely due to a slight deviation of the structure height of the phase grating from its nominal value. Further, the peak visibility in the measurement is lower than the peak visibility of the simulation, which is most likely due to non-ideal source and (a)

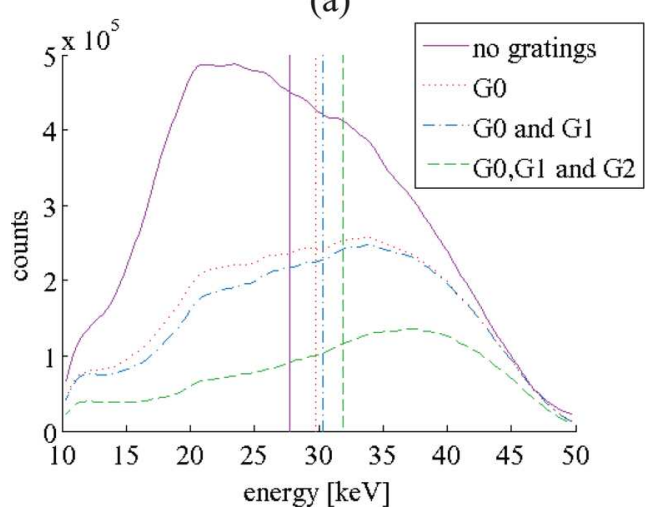

(b)

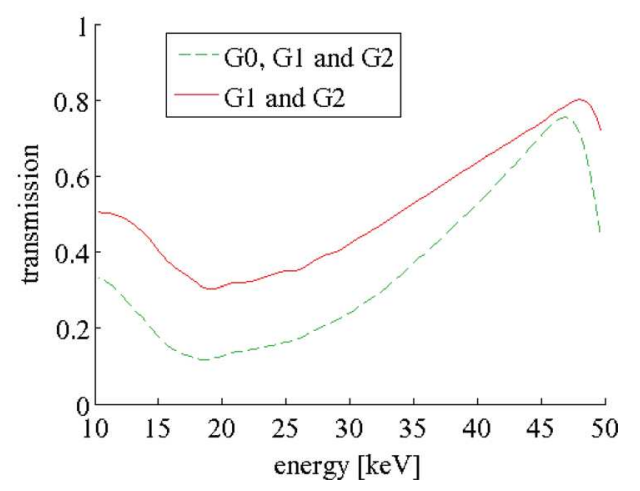

FIG. 1. (a) Measured energy spectra for different configurations of the GI, acquired by using a threshold sweep scan with an exposure time of $5 \mathrm{~s}$ per sweep step. The mean energies are marked with the vertical lines. (b) Energy resolved X-ray transmission through all gratings (solid line) and through G1 and G2 only (dashed line). For both curves, the lowest transmission (highest absorption) is at around $18 \mathrm{keV}$. 
(a)

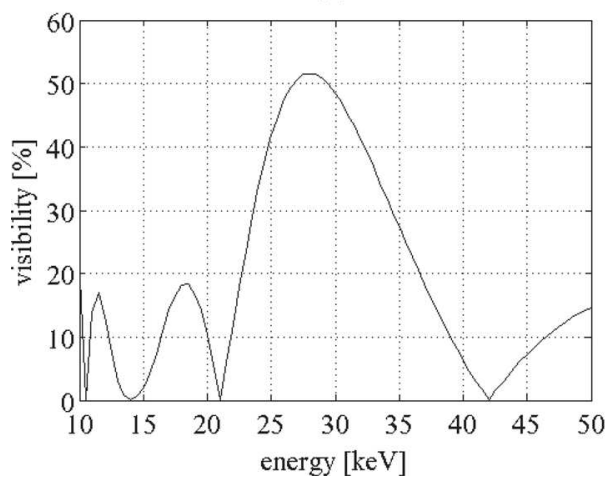

(b)

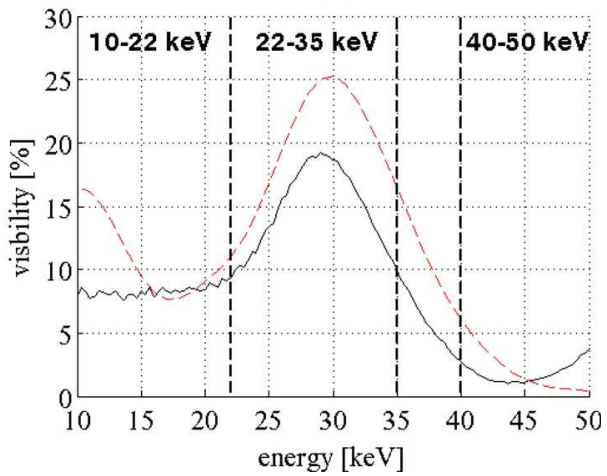

FIG. 2. (a) Theoretical visibility as a function of energy for a perfect GI with ideal absorption gratings. (b) Measured (solid line) and simulated (dashed line) visibility as a function of X-ray energy on the experimental interferometer. For the simulation, an energy bandwidth of $3 \mathrm{keV}$ (standard deviation) has been used, which approximately corresponds to the energy resolution of the detector. The dashed vertical lines indicate the bandwidth selections for the images in Fig. 3. grating qualities. The lower and upper sidelobes of the visibility curve located between 18 and $21 \mathrm{keV}$ and between 40 and $50 \mathrm{keV}$, which are visible in the theoretical plot of Fig. 2(a), could not be resolved in the measurement. For the upper side lobe, this can be explained by the reduced efficiency of the absorption gratings G0 and G2, decreasing the visibility. For the lower sidelobe, which has a width of only $5 \mathrm{keV}$, it is most likely the limited energy resolution by the detector which leads to a blurring of this sidelobe, averaging out the visibility in this region. At the very end of the high energy range (close to $50 \mathrm{keV}$ ), the measured visibility curve in Fig. 2(b) shows a tail of slightly increased visibility, which occurs due to low counts in the bins (low flux and narrow bins). The number of counts in such a bin is close to zero or may happen to be even negative, which erroneously leads to an increased measured visibility.

Once the spectral acceptance of a GI is known, an energy resolving detector offers the possibility to optimize the performance of the imaging system by selecting a custom energy bandwidth. In particular for DPC images, where the visibility and, thus, the SNR (Eq. (1)) is strongly dependent on the energy, an appropriate bandwidth selection can significantly improve the image quality. The effect of such an energy band selection in absorption and phase contrast imaging is illustrated in Fig. 3. It shows a tomographic slice of a phantom, reconstructed from 100 projections, in phase and absorption contrast. Eight phase steps were acquired with an exposure time of $2 \mathrm{~s}$ per step. The quantitative reconstruction shows the components of the complex refractive index $n=1-\delta+i \beta$, where $\delta$ represents the phase and $\beta$ the attenuation contrast, respectively. The cylindrical PMMA phantom has a diameter of $30 \mathrm{~mm}$ and contains hollow rods (rod diameters from 0.7 to $1.2 \mathrm{~mm}$ ), which are filled with water (marked with arrow). Although the resolution is low, the rods can clearly be distinguished from water in the phase image. Due to the nearly identical attenuation factors ( $\beta$ values) of PMMA and water, the rods are invisible in the absorption images. Some of the rods were not filled with water and appear as dark spots in the absorption and phase image (marked with arrow). The images illustrate the
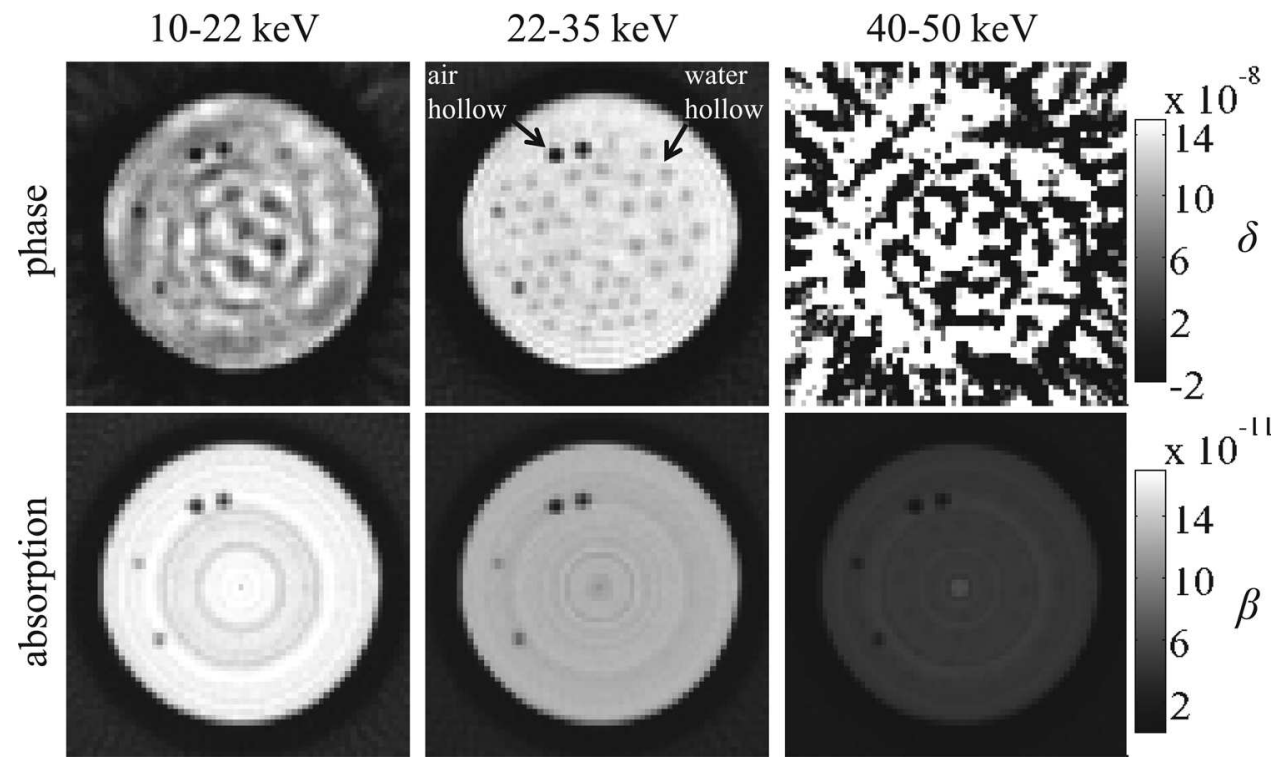

FIG. 3. Tomographic slice of a phantom in phase and absorption contrast, reconstructed in different energy windows, which are also marked in Fig. 2(b). The phantom is made of PMMA and contains hollow rods filled with water. Some of the rods were not filled with water and appear as dark spots in the slice (see arrows). The rods filled with water can be resolved in the phase contrast image, but not in the absorption, which is due to the low difference between the attenuation coefficients of PMMA and water. The phase contrast images in the first row show the strong dependency of the SNR on the selection of the energy window. Each window has a different mean visibility (see Fig. 2), which is the reason why the phase image in the middle row (22-35 keV) has low noise while the images in the left and right rows (10-22 keV and $40-50 \mathrm{keV}$, respectively) are strongly distorted by noise. This effect is not observable in the absorption images, as their noise properties are independent on the visibility. 


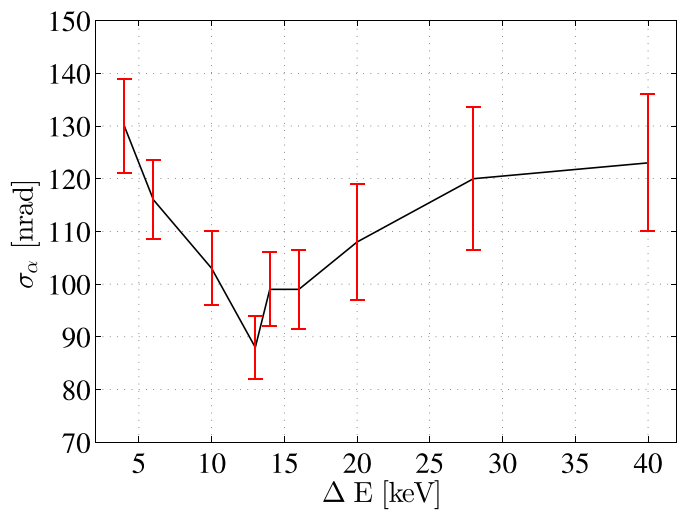

FIG. 4. Standard deviation of the noise $\left(\sigma_{\alpha}\right)$ in the refraction angle measurement (or DPC image) as a function of energy bandwidth around the design energy. The noise is minimized if the energy bandwidth is $13 \mathrm{keV}$. The error bars indicate the uncertainty (standard deviation) of $\sigma_{\alpha}$.

dependency of the SNR on the selection of the energy band. In the absorption images ( $\beta$ values), the signal is proportional to $1 / E^{4}$ and, therefore, a significantly decreased absorption signal (lower contrast versus surrounding air) can be observed in the higher energy windows. Since noise is proportional to $1 / \sqrt{N}$ and thus completely independent on the visibility, it does not change significantly in the different windows. On the other hand, in the phase image, where noise is predominantly determined by the visibility $V$ (see Eq. (1)), the SNR is strongly dependent on the selected energy window. Since the middle window has been chosen according to the spectral acceptance of the GI, noise is minimized there. In the upper energy window, the image is completely distorted by noise due to the low visibility.

According to Eq. (1), the sensitivity of phase contrast images is mainly dependent on the visibility $V$ and the number of photons $N$. In an imaging experiment with a polychromatic spectrum, $V$ and $N$ are usually counteracting, in the sense that a high bandwidth normally yields high flux but low visibility, whereas a narrow bandwidth increases the visibility but significantly reduces the flux. Therefore, there must be an optimum for the spectral bandwidth, which is likely to be located around the design energy of the GI and which minimizes the noise. In order to find this optimum, the noise standard deviation of DPC images, $\sigma_{\alpha}$, has been measured from a set of DPC projections without an object for different bandwidths around the design energy (28 keV). The result is displayed in Fig. 4 and indicates that an appropriate bandwidth selection minimizes the noise. For the setup in this experiment, highest performance (lowest noise) was obtained for a bandwidth of $13 \mathrm{keV}$ (spectrum between 22 and $35 \mathrm{keV}$ ).

This result contains important information for specific applications. For instance in applications where high flux is important, a high X-ray tube voltage combined with an optimized bandwidth selection would maximize the sensitivity per time. As opposed to conventional filtering, a bandwidth selection with an energy resolving detector provides the great possibility to selectively remove parts of the spectrum, while desired parts are fully transmitted. On the other hand, for an application which requires low dose, the optimization of the bandwidth could be used to determine the appropriate filters.

In summary, the impact of energy resolved detection for phase contrast imaging with a GI has been demonstrated. The ideal efficiency of the sensor and the adjustable energy thresholds are the key features to access full information about the X-ray spectrum in grating interferometry. X-ray spectra, flux reduction or the intrinsic dose increase of the GI can be experimentally quantified with high accuracy. Due to the strong dependency of the sensitivity of phase contrast images on the X-ray spectrum, an appropriate bandwidth selection with an energy resolving detector can significantly improve the sensitivity per time or provide the relevant information to minimize dose.

We thank Gordan Mikuljan from Paul Scherrer Institut for his support on the mechanical design. Part of this work has been supported by the ERC Grant No. ERC-2012-StG 310005-PhaseX.

${ }^{1}$ R. Fitzgerald, Phys. Today 53(7), 23 (2000).

${ }^{2}$ F. Pfeiffer, M. Bech, O. Bunk, P. Kraft, E. Eikenberry, C. Brönnimann, C. Grünzweig, and C. David, Nature Mater. 7, 134 (2008).

${ }^{3}$ A. Momose and J. Fukuda, Med. Phys. 22, 375 (1995).

${ }^{4}$ S. A. McDonald, F. Marone, C. Hintermuller, G. Mikuljan, C. David, F. Pfeiffer, M. Stampanoni, and C. Hintermüller, J. Synchrotron Radiat. 16, 562 (2009).

${ }^{5}$ F. Pfeiffer, T. Weitkamp, O. Bunk, and C. David, Nat. Phys. 2, 258 (2006).

${ }^{6}$ M. Engelhardt, J. Baumann, M. Schuster, C. Kottler, F. Pfeiffer, O. Bunk, and C. David, Appl. Phys. Lett. 90, 224101 (2007).

${ }^{7}$ T. Thüring, P. Modregger, T. Grund, J. Kenntner, C. David, and M. Stampanoni, Appl. Phys. Lett. 99, 041111 (2011).

${ }^{8}$ A. Tapfer, M. Bech, A. Velroyen, J. Meiser, J. Mohr, M. Walter, J. Schulz, B. Pauwels, P. Bruyndonckx, X. Liu, A. Sasov, and F. Pfeiffer, Proc. Natl. Acad. Sci. U.S.A. 109, 15691 (2012).

${ }^{9}$ J. Iwanczyk, E. Nygard, J. Wessel, N. Malakhov, G. Wawrzyniak, N. Hartsough, T. Gandhi, and W. Barber, in IEEE Nuclear Science Symposium (2011), pp. 4745-4750.

${ }^{10}$ R. Raupach and T. Flohr, Med. Phys. 39, 4761 (2012).

${ }^{11}$ E. Roessl, T. Koehler, U. van Stevendaal, G. Martens, N. Hauser, Z. Wang, and M. Stampanoni, Proc. SPIE 8313, 831354 (2012).

${ }^{12}$ T. Weitkamp, A. Diaz, C. David, F. Pfeiffer, M. Stampanoni, P. Cloetens, and E. Ziegler, Opt. Express 13, 6296 (2005).

${ }^{13}$ V. Revol, C. Kottler, R. Kaufmann, U. Straumann, and C. Urban, Rev. Sci. Instrum. 81, 073709 (2010).

${ }^{14}$ M. Engelhardt, C. Kottler, O. Bunk, C. David, C. Schroer, J. Baumann, M. Schuster, and F. Pfeiffer, J. Microsc. 232, 145 (2008). 